\title{
Propagation of energetic electrons through the solar corona and the interplanetary medium
}

\author{
H. Önel ${ }^{1,2}$, G. Mann ${ }^{1}$, and E. Sedlmayr ${ }^{2}$ \\ 1 Astrophysical Institute Potsdam (AIP), An der Sternwarte 16, 14482 Potsdam, Germany \\ e-mail: honel@aip.de \\ 2 University of Technology Berlin, Centre of Astronomy and Astrophysics, Hardenbergstraße 36, 10623 Berlin, Germany
}

Received 20 March 2006 / Accepted 26 October 2006

\section{ABSTRACT}

\begin{abstract}
Aims. The electron transport is investigated numerically after an electron transport model is deduced.
Methods. This model for electron propagation considers global electric and magnetic fields, as well as local Coulomb collisions. A new way to handle the electron's pitch angle evolution by using a binary dice within the treatment of the Coulomb scattering is introduced. The conditions in the solar plasma are represented by average and commonly used models, such that numerical simulations can be performed easily.

Results. The model for electron propagation finally obtained makes it possible to investigate how the Coulomb collisions act on the pitch angle, while electrons are transported through the solar plasma. Some chosen numerical results for different initial conditions are presented in the paper.
\end{abstract}

Key words. Sun: particle emission - Sun: flares - Sun: magnetic fields - Sun: atmosphere - X-rays: bursts - Sun: radio radiation

\section{Introduction}

In solar flares a large amount of energy is released in the form of particles and enhanced emissions of radiation from the radio up to the $\gamma$-ray regions. Energetic electrons accelerated in these events are of special interest, since they are considered to be responsible for the observable non-thermal radio and X-ray radiations (Lin 1974). It is generally accepted that these electrons are accelerated up to high energies in all directions along the magnetic field in the corona, triggered by the process of magnetic reconnection.

After these energetic electrons are generated in the corona at their acceleration site, they travel towards the dense chromosphere, and if they have sufficient energy they can produce X-ray emission via bremsstrahlung (Brown 1972). On the other hand, there are also electrons travelling away from the Sun, namely towards the higher corona, and possibly into the interplanetary space, if they meet open field lines. These electrons may reach the Earth within a few minutes (Lin 1974). There they can be studied by in situ measurements (e.g., by the WIND spacecraft).

Figure 1 shows the energetic electrons leaving the acceleration site in the corona. Fast electrons passing through the solar plasma cause radio signatures via beam-plasma instabilities (see Melrose 1985). The electrons directed outwards excite type III radio emission. It is possible to observe and to study these electrons by radio observations. Figure 2 presents a dynamic radio spectrum from March 11, 1999 (Önel et al. 2005, 2006) obtained by the Radio Sweep Spectropolarimeter (Mann et al. 1992). The frequencies are recorded in the range from $110 \mathrm{MHz}$ to $400 \mathrm{MHz}$ and are plotted versus the time period of 8:18:50 UT to 8:19:05 UT. This spectrum contains one type III burst originating at $370 \mathrm{MHz}$ and 8:19:51.5 UT. It also contains a second faint type III burst starting at $320 \mathrm{MHz}$ level at

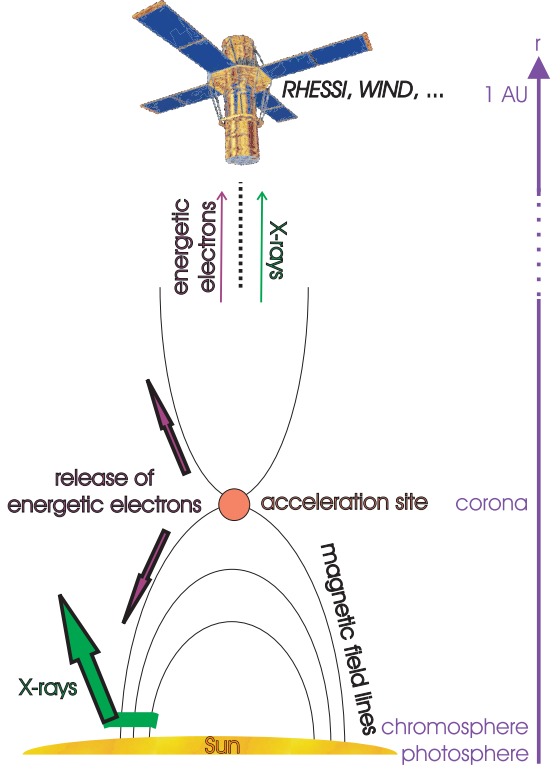

Fig. 1. Schematic illustration of propagating energetic electrons towards the Sun's surface and in the interplanetary medium generated at the acceleration site.

8:19:55.5 UT, which is superimposed by a type U burst. While the type III bursts are generated by fast electrons drifting outward along open field lines, the type $U$ bursts are assumed to be created by fast drifting electrons in closed magnetic field structures (see Yokoyama et al. 2002). In such a case, the electrons also produce radio continuum radiation in terms of so-called type IV radio bursts (see, e.g., Robinson 1985, as a review). 


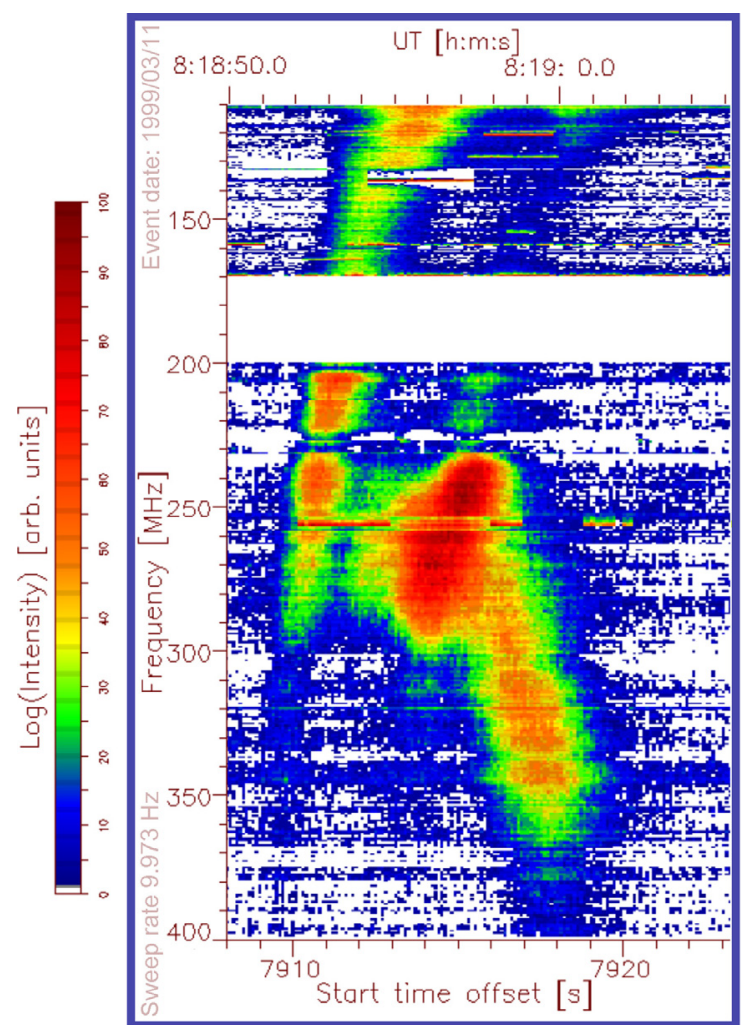

Fig. 2. Dynamical radio spectrum of an event from March 11, 1999 obtained by the Radio Sweep Spectropolarimeter at the Astrophysical Institute Potsdam (AIP) in Germany. The frequency is shown on a reversed axis in dependence on time, whereas the radiation intensities are colour coded.

In 2002, NASA launched RHESSI the Ramaty High Energy Solar Spectroscopic Imager (Lin et al. 2002), which is designed to observe the hard X- and $\gamma$-ray radiation emitted by the Sun with a high temporal, spatial, and energy resolution. One of RHESSI's main mission objectives is to provide information on the particle acceleration processes during solar flares. Since RHESSI can only observe hard X-and $\gamma$-ray radiation from the Sun, it can only indirectly provide information about the acceleration process itself (Brown 1971). Therefore, a model for particle propagation is needed to acquire information about the particle acceleration site, which is in general different from the radiation emission site.

In this paper, a model for the propagation of a test electron through the solar plasma is studied. The journey of such a test electron through the solar plasma is influenced by the global magnetic and electric fields as well as by local Coulomb collisions. These influences are investigated in a quantitative manner, both separately and jointly. The propagating electrons are also affected by the local plasma waves via wave-particle interaction (e.g., Kunow et al. 1991), which is not taken into account in the present study: In the solar corona the particle number density and the collision frequency are quite high. In addition, the magnetic field diverges much more than the interplanetary field. Therefore the wave particle interactions only have a little influence on the electron propagation in the solar plasma. Whenever needed, the model presented in this paper can easily be applied to proton or ion propagations with minor tuning only.

In Sect. 2 the set of equations regarding the electron motion in the solar plasma is derived. Next, in Sect. 3 the plasma background, i.e., the spatial behaviour of the particle density,

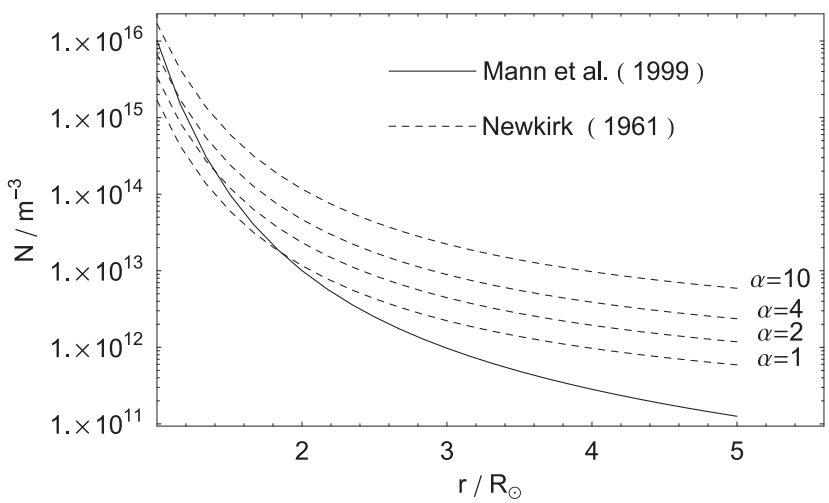

Fig. 3. Total particle density is shown as a function of heliocentric distance.

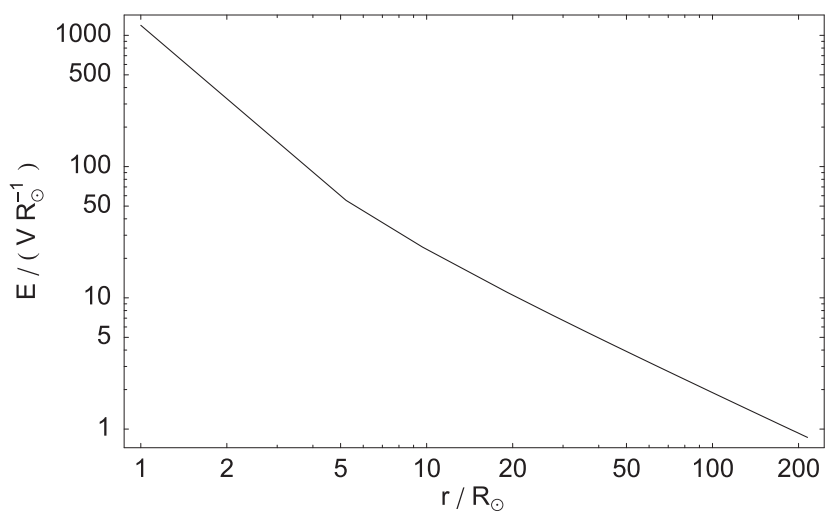

Fig. 4. The global model for the electric field based on Eqs. (22) and (25) is shown.

the electric field, and magnetic field are introduced under coronal and interplanetary conditions. The results are presented and discussed in Sect. 4, which is then followed by a summary of the main issues in Sect. 5.

\section{Model for electron propagation}

In the following section the set of equations of motion for a test electron is derived to describe its motion away from the acceleration site. As already stated earlier, the influence of the electric and magnetic fields, as well as the Coulomb collisions, are taken into account.

\subsection{Effects of the magnetic and electric fields}

Initially the effects of Coulomb collisions are neglected to study only the pure influence of the magnetic and electric field on the propagation of a test electron through the coronal and interplanetary plasma. The frictionless motion of a test electron on which a general force $\boldsymbol{F}$ and a magnetic flux density $\boldsymbol{B}$ acts can be described by the most general equation of motion

$$
\frac{\mathrm{d} \boldsymbol{u}}{\mathrm{d} t}=\frac{-e}{m_{\mathrm{e}}}\left(\frac{1}{-e} \boldsymbol{F}+\boldsymbol{u} \times \boldsymbol{B}\right) .
$$

Here $e, m_{\mathrm{e}}, \boldsymbol{u}$, and $\boldsymbol{B}$ represent the elementary charge, the mass of the electron, the electron velocity, and the magnetic flux density, respectively. Since the gravitational force $\boldsymbol{F}_{\mathrm{G}}$ is neglected due to the small electron mass in this paper, the force $\boldsymbol{F}=\boldsymbol{F}_{\mathrm{G}}+\boldsymbol{F}_{\mathrm{E}} \approx \boldsymbol{F}_{\mathrm{E}}$ considers only the electric force $\left(\boldsymbol{F}_{\mathrm{E}}=-e \boldsymbol{E}\right)$, which follows from the electric field $\boldsymbol{E}$. 
Only electrons with initial energies up to $50 \mathrm{keV}$ are regarded in this paper. Thus, Eq. (1) describes the electron motion in a non relativistic manner and the deviation of Eq. (1) due to relativistic effects is only of the order of $12 \%$. Furthermore, the global electric and magnetic fields are considered to be aligned with each other. In addition, the magnetic field is assumed to have only small spatial variations, i.e., it fulfils the relationship

$\mathcal{B}=\left|B^{-1}\left(\boldsymbol{r}_{\text {Larmor }} \cdot \nabla\right) \boldsymbol{B}\right| \ll 1$,

where the Larmor radius $r_{\text {Larmor }}$ is defined by

$r_{\text {Larmor }}=\frac{m_{\mathrm{e}}}{e B} u \sin [\Theta]$.

The quantity $\Theta=\angle(\boldsymbol{B}, \boldsymbol{u})$ represents the pitch angle. In such a case the magnetic moment $\mu$ given by

$\mu=\frac{m_{\mathrm{e}}}{2 B} u_{\perp}^{2}$

turns out to be an adiabatic constant of motion (see Kegel 1998; Treumann \& Baumjohann 1999, Chap. 2 in both books), i.e.,

$0=\frac{\mathrm{d} \mu}{\mathrm{d} r}=\frac{1}{2} \frac{\mathrm{d}}{\mathrm{d} r}\left(\frac{m_{\mathrm{e}}}{B} u_{\perp}^{2}\right)$.

$u_{\perp}=u \sin [\Theta]$ in Eq. (4) denotes the perpendicular component of the relative velocity $\boldsymbol{u}$ referring to the magnetic field direction. The quantity $r$ represents a distance along the magnetic field.

The 3-dimensional problem described by Eq. (1) can be treated in a 2-dimensional manner, if Alfvén's gyro centre approximation and Eq. (5) are used. Then the particle propagation can be separated into two different motions, namely into the drift motion (i.e., motion that is parallel to the magnetic field), and into the gyro motion (Larmor motion, i.e., motion that is perpendicular to the magnetic field), as follows:

$$
\begin{aligned}
\frac{\mathrm{d} u}{\mathrm{~d} t} & =\frac{F}{m_{\mathrm{e}}} \cos [\Theta] \\
\frac{\mathrm{d} \Theta}{\mathrm{d} t} & =-\frac{\sin [\Theta]}{u} \frac{F}{m_{\mathrm{e}}}+\frac{u \sin [\Theta]}{2 B} \frac{\mathrm{d} B}{\mathrm{~d} r} .
\end{aligned}
$$

Equations (6) and (7) are the deterministic equations for the temporal evolution of the test particle's velocity and pitch angle (Bai 1982). The quantity $\mathrm{d} r$ represents the distance along the magnetic field, which the electron traverses within the time period $\mathrm{d} t$.

\subsection{Effect of Coulomb collisions/friction force}

A test electron that propagates through a certain plasma background of one particle species (with charge $q$, mass $M$, and particle density $N$ ) is decelerated due to Coulomb collisions (Chap. 13, p. 652 in Jackson 1975; Estel \& Mann 1999) as described by

$\frac{\mathrm{d} u}{\mathrm{~d} t}=-\frac{e^{2} \operatorname{sign}[u]}{4 \pi \epsilon_{0}^{2}} \frac{q^{2} N}{\chi^{2} u^{2}} \ln \left[\sqrt{\frac{\lambda_{\mathrm{D}}^{2}+b_{0}^{2}}{2 b_{0}^{2}}}\right]$,

where

$\chi=\left(\frac{1}{m_{\mathrm{e}}}+\frac{1}{M}\right)^{-1}$

stands for the reduced mass between the test electron and the plasma particle of the atmosphere. The other appearing

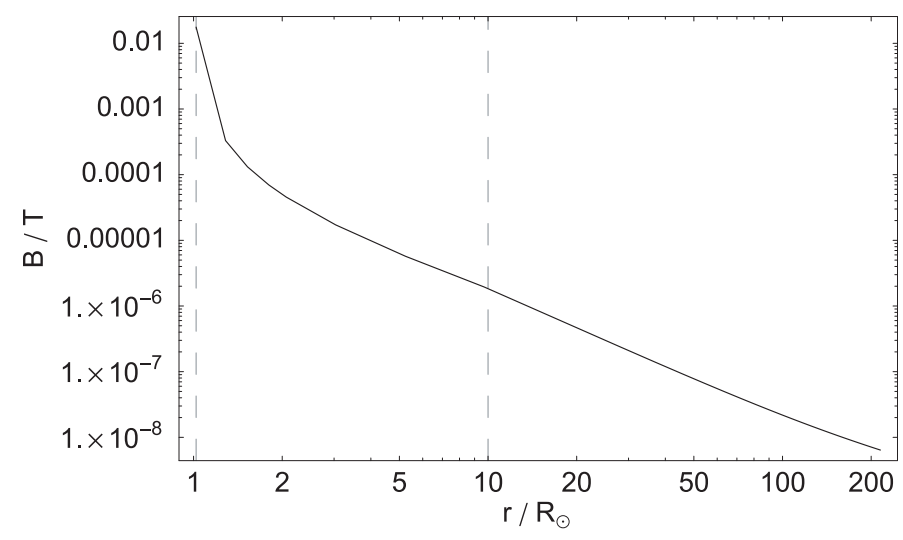

Fig. 5. The global model for the magnetic flux density according to Eq. (29) is shown in dependence on the heliocentric distance in units of the solar radius. The heliocentric radii $1.02 R_{\odot}$ and $10 R_{\odot}$ are marked with vertical dashed lines.

quantities are the Debye length $\lambda_{\mathrm{D}}=\left(\epsilon_{0} k_{\mathrm{B}} T\left(N_{\mathrm{e}} e^{2}\right)^{-1}\right)^{0.5}$ (see Treumann \& Baumjohann 1997, Chap. 1, p. 1), and the impact parameter $b_{0}=e|q|\left(4 \pi \epsilon_{0} \chi u^{2}\right)^{-1}$ (see Goldston \& Rutherford 1995 , Chap. 11 , p. 166), which leads to a $90^{\circ}$ deflection of the electron, when it collides with the plasma particle. $N_{\mathrm{e}}$ represents the electron particle density in the plasma, whereas $T$ is the atmosphere's plasma temperature. The quantities $\epsilon_{0}$ and $k_{\mathrm{B}}$ are the permittivity of free space, and the Boltzmann constant, respectively. The sign function in Eq. (8) comes from the convention used: a test electron moving towards the Sun (inward propagation) fulfils $u<0$, whereas $u>0$ means that the test electron is propagating outward.

So far all equations have been deterministic, but this changes if the Coulomb collisions are considered correctly, as it is explained in the following. The mean scattering angle $\bar{\theta}$ vanishes due to the symmetry of the scattering process. That is expected if the test particle is scattered randomly without any preferred direction at each collision. However, the mean squared scattering angle $\overline{\theta^{2}}$ does not vanish in general. It follows that it is

$\frac{\mathrm{d} \bar{\theta}^{2}}{\mathrm{~d} t} \approx\left|\frac{e^{2} q^{2} N}{2 \pi \epsilon_{0}^{2} \chi^{2} u^{3}} \ln \left[\sqrt{\frac{\lambda_{\mathrm{D}}^{2}+b_{0}^{2}}{2 b_{0}^{2}}}\right]\right|$,

as explained in Appendix A.

Even though many equally distributed scattering processes do not affect the pitch angle $(\bar{\theta}=0)$, each single Coulomb collision does. Each collision deflects the test electron either in one or in the other scattering direction with the same probability, which has to be considered within the general equation of motion when solving iteratively. Hence after one time step $\mathrm{d} t$, the pitch angle changes with respect to Coulomb collisions as

$\Theta[t+\mathrm{d} t]:=\Theta[t]+\left.\mathcal{P} \frac{\mathrm{d} \theta_{\mathrm{C}}}{\mathrm{d} t}\right|_{t, \Theta[t]} \mathrm{d} t$,

where $\mathcal{P}$ denotes the result of a binary dice, which can be either -1 or 1 . The quantity $\mathrm{d} \theta_{\mathrm{C}}$ is defined by the total derivative of the root mean square of $\theta$, namely $\mathrm{d} \theta_{\mathrm{C}}:=\sqrt{\mathrm{d} \bar{\theta}^{2}}$. In summary, Eqs. (8) and (11) describe the influence of the Coulomb collisions on the propagation of a test electron.

\subsection{Final equations of motion}

After deriving the tools needed to describe the transport of a test electron within the solar plasma, the equations of motion 

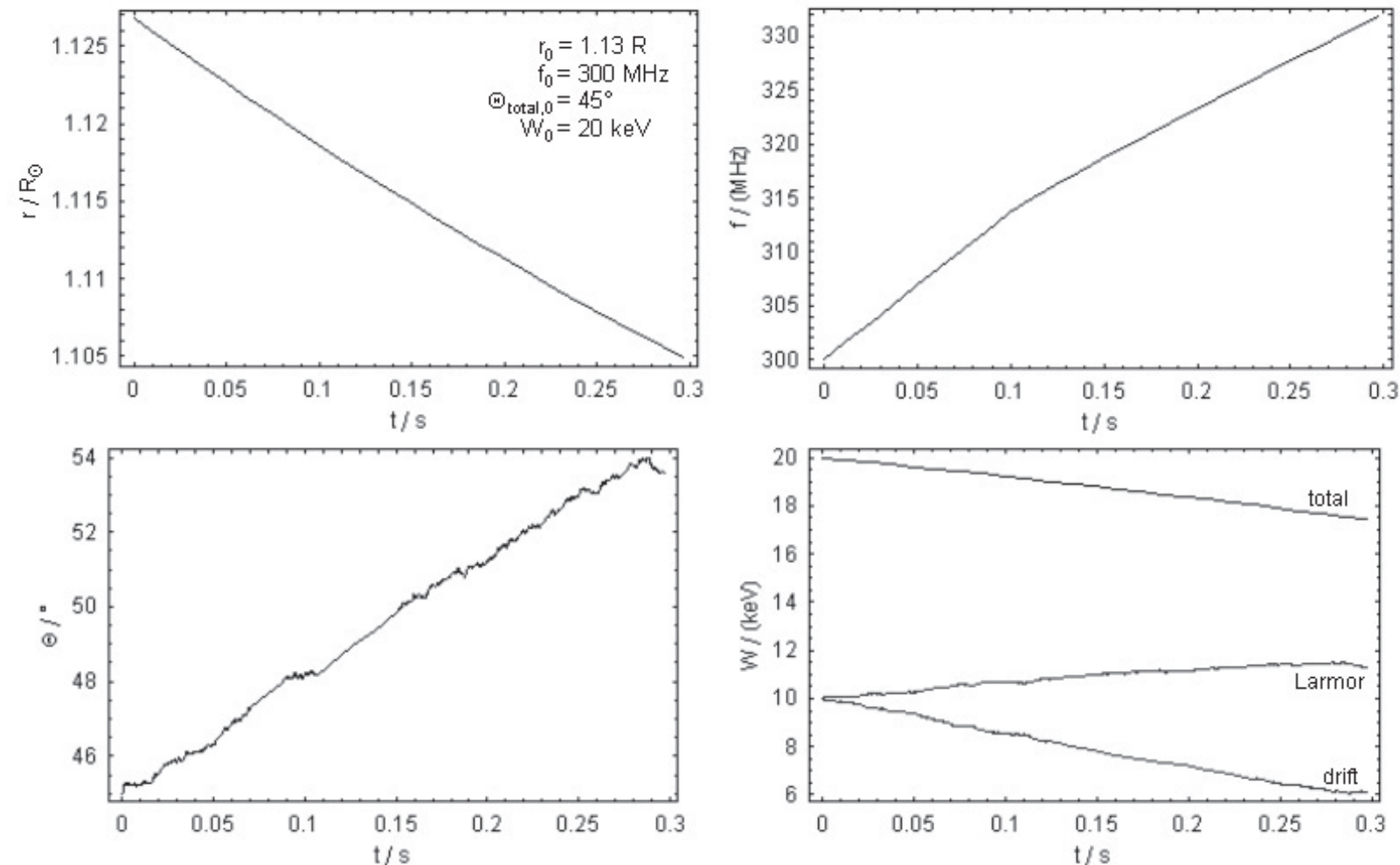

Fig. 6. Arithmetically averaged outer movement of 1000 test electrons. Top left: the averaged altitude is plotted in dependence on time $r[t]$. Top right: the averaged electron plasma frequency is plotted in dependence on time $f[t]$. Bottom left: the averaged pitch angle is plotted in dependence on time $\Theta[t]:=\Theta_{\text {total }}[t]$. Bottom right: the averaged energy is plotted in dependence on time $W[t]$.
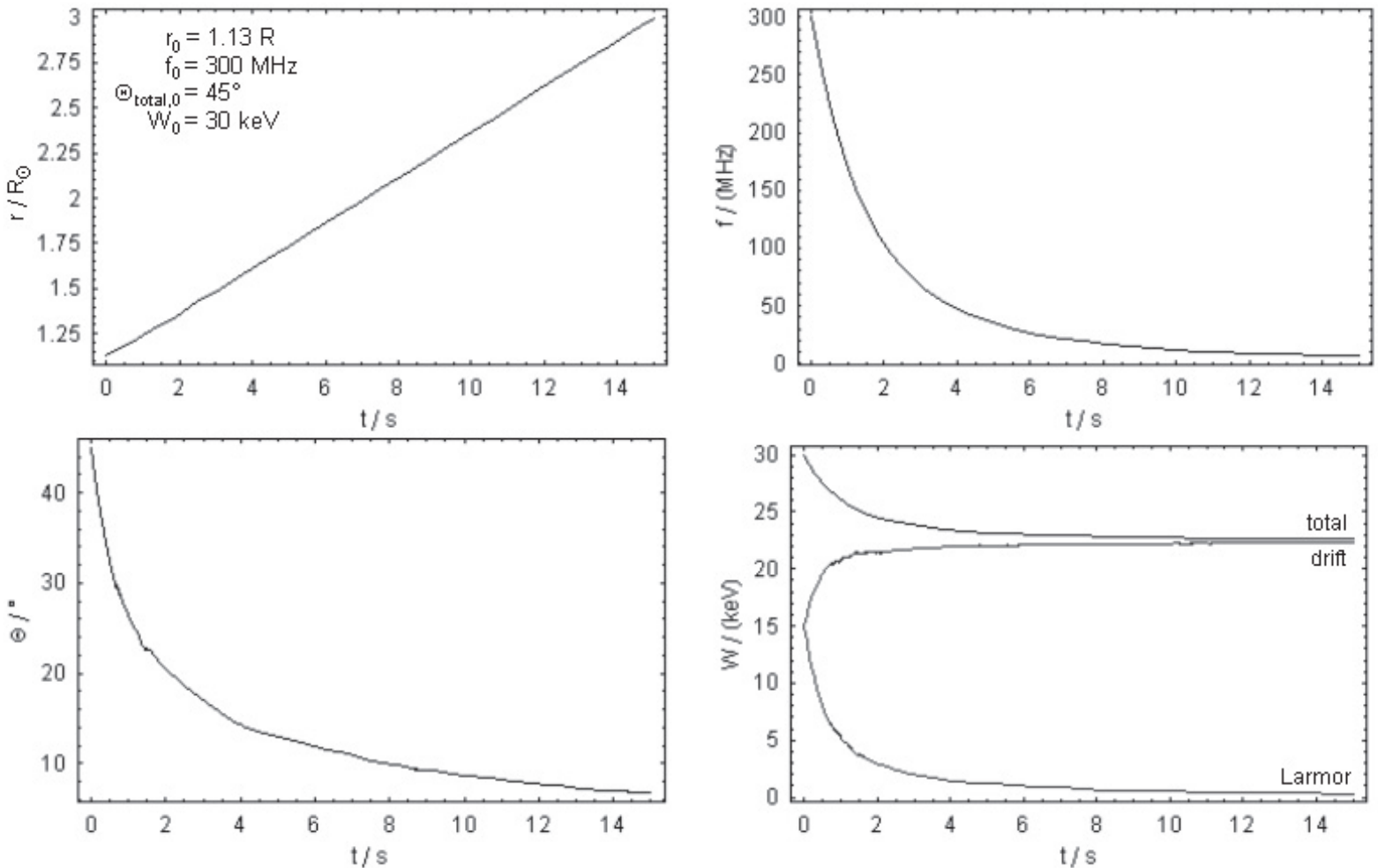

Fig. 7. Arithmetically averaged outer movement of 1000 test electrons. Top left: the averaged altitude is plotted in dependence on time $r[t]$. Top right: the averaged electron plasma frequency is plotted in dependence on time $f[t]$. Bottom left: the averaged pitch angle is plotted in dependence on time $\Theta[t]:=\Theta_{\text {total }}[t]$. Bottom right: the averaged energy is plotted in dependence on time $W[t]$. 

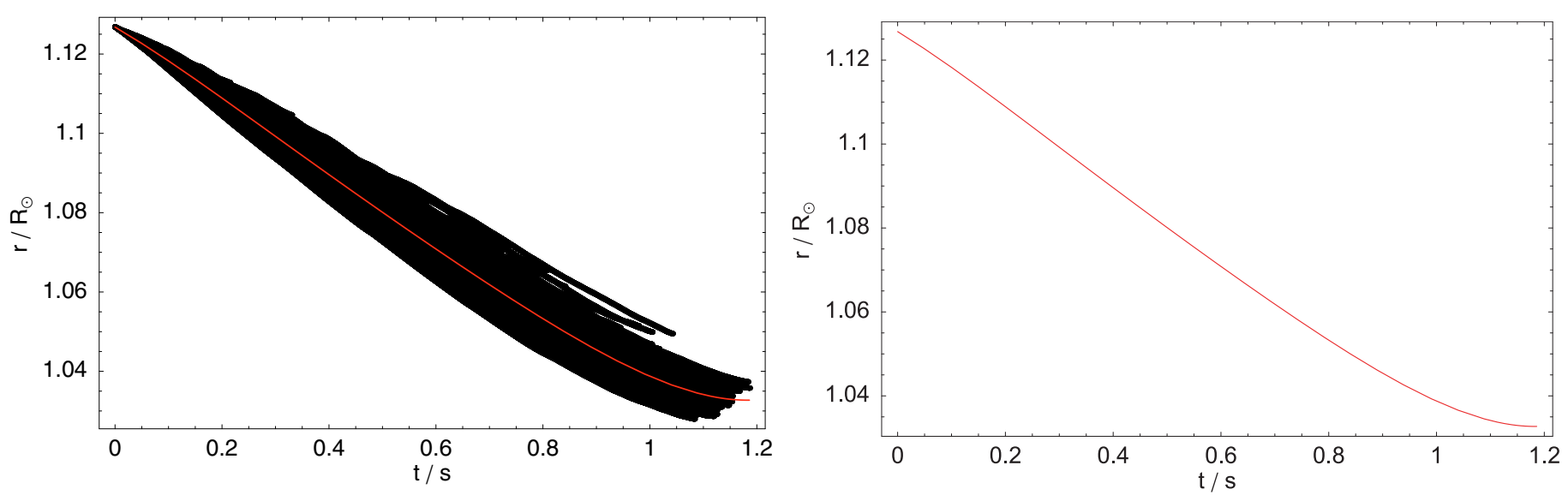

Fig. 8. On the left hand-side the random walk of 1000 electrons is illustrated. All electrons start their motion with the same initial conditions: initial heliocentric radial distance of about $1.13 R_{\odot}$, initial pitch angle of $45^{\circ}$, initial energy of $20 \mathrm{keV}$. The red line, which is also plotted on the right-hand side as a single diagram, shows the best fit of all these random trajectories according to the minimum square deviation.
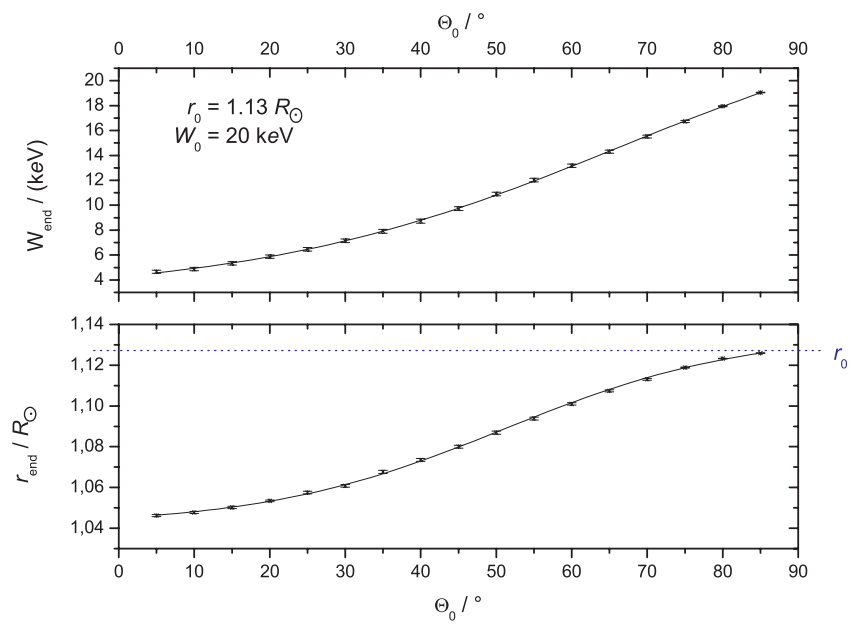

Fig. 9. Influence of the initial pitch angle for the inner transport process. Top: The electron's kinetic energy is illustrated at the point $u_{\|}=0$ depending on its initial pitch angle. Bottom: the heliocentric electron distance is plotted at the point $u_{\|}=0$ depending on its initial pitch angle.

are going to be derived considering the magnetic field, the electric field, and the Coulomb collisions. Furthermore the assumption that the atmospheric plasma does not consist of one particle species only (see Sect. 3) is made, and the total velocity $u_{\text {total }}$ is introduced. The index "total" means that the collisional effects of all particle species are included. Note that in the final equations of motion derived in the current section, the global magnetic and electric fields in a deterministic, and the Coulomb interaction in a stochastic manner are taken into consideration.

For the total velocity,

$\frac{\mathrm{d} u_{\text {total }}}{\mathrm{d} t}=\frac{e|E| \operatorname{sign}\left[u_{\text {total }}\right]}{\chi_{\text {total }}} \cos \left[\Theta_{\text {total }}\right]+\frac{\mathrm{d} u_{\mathrm{C}, \text { total }}}{\mathrm{d} t}$

is obtained. The Coulomb interaction is taken into account in the last term of Eq. (12), as defined by

$\frac{\mathrm{d} u_{\mathrm{C}, \text { total }}}{\mathrm{d} t}:=\frac{\mathrm{d} u_{\mathrm{e}}}{\mathrm{d} t}+\frac{\mathrm{d} u_{\mathrm{p}}}{\mathrm{d} t}+\frac{\mathrm{d} u_{\mathrm{He}^{2+}}}{\mathrm{d} t}$

where each addend on the right-hand side follows from Eq. (8) for the correct particle species, i.e., here in the plasma electrons (index "e"), protons (index "p"), and fully ionised helium atoms (index " $\left.\mathrm{He}^{2+} "\right)$ are considered. Analogously

$$
\frac{\mathrm{d} \theta_{\mathrm{C}, \text { total }}}{\mathrm{d} t}:=\frac{\mathrm{d} \theta_{\mathrm{e}}}{\mathrm{d} t}+\frac{\mathrm{d} \theta_{\mathrm{p}}}{\mathrm{d} t}+\frac{\mathrm{d} \theta_{\mathrm{He}^{2+}}}{\mathrm{d} t}
$$

is defined. Equation (14) considers the resulting different scattering angles due to the electron's collision with the different particle species. From Eq. (11) the quantity $\Theta_{\text {total }}$ is obtained, if $\frac{\mathrm{d} \theta_{\mathrm{C}}}{\mathrm{d} t}$ is substituted with $\frac{\mathrm{d} \theta_{\text {C.total }}}{\mathrm{d} t}$. Finally, the change of the radial component $r$ of the electron's motion is described with

$\frac{\mathrm{d} r}{\mathrm{~d} t}=u_{\text {total }} \cos \left[\Theta_{\mathrm{total}}\right]=: u_{\mathrm{total}, \|}$.

Note that the radial component is aligned along the global magnetic field and $r$ represents the distance between the test electron and the centre of the Sun.

In summary, if the initial altitude $r_{0}:=r\left[t_{0}\right]$, the initial velocity $u_{\text {total }, 0}:=u_{\text {total }}\left[t_{0}\right]$, and the initial pitch angle $\Theta_{\text {total }, 0}:=\Theta_{\text {total }}\left[t_{0}\right]$ are known, it is possible to calculate the propagation of an electron away from the acceleration site with the following final equations of motion:

$$
\begin{aligned}
r[t+\mathrm{d} t] & =u_{\text {total }}[t] \cos \left[\Theta_{\text {total }}[t]\right] \mathrm{d} t \\
u_{\text {total }}[t+\mathrm{d} t] & =u_{\text {total }}[t]+\left.\frac{\mathrm{d} u_{\text {total }}}{\mathrm{d} t}\right|_{t, \Theta_{\text {total }}[t]} \mathrm{d} t \\
\Theta_{\text {total }}[t+\mathrm{d} t] & =\Theta_{\text {total }}[t]+\left.\left(\frac{\mathrm{d} \Theta_{\text {total }}}{\mathrm{d} t}+\mathcal{P} \frac{\mathrm{d} \theta_{\mathrm{C}, \text { total }}}{\mathrm{d} t}\right)\right|_{t, \Theta_{\text {total }}[t]} \mathrm{d} t .
\end{aligned}
$$

The quantity of $\frac{\mathrm{d} \Theta_{\text {total }}}{\mathrm{d} t}$ in Eq. (18) only describes the change of the pitch angle in accordance to the magnetic and electric field effects as described by Eq. (7). The obtained Eqs. (16) to (18) can provide information about the acceleration site by observing electrons and/or by electron radiated emissions far away from the acceleration site, as they allow a so-called backward calculation $(\mathrm{d} t<0)$ : By assuming that the particles for the inner and the outer motion are simultaneously accelerated from the same acceleration site, one can estimate both the altitude and the energies of the electrons at the acceleration site $\left(t=0=: t_{0}\right)$, if for the inner and outer particle motion at any $t_{\text {end }}>t_{0}$ the altitude $r\left[t_{\text {end }}\right]$, the velocity $u_{\text {total }}\left[t_{\text {end }}\right]$, and the pitch angle $\Theta_{\text {total }}\left[t_{\text {end }}\right]$ are known. To study the influence of the global magnetic and electric fields on the propagation of a test electron from its acceleration site through the coronal and interplanetary plasma, Eqs. (16) to (18) have to be numerically solved. 


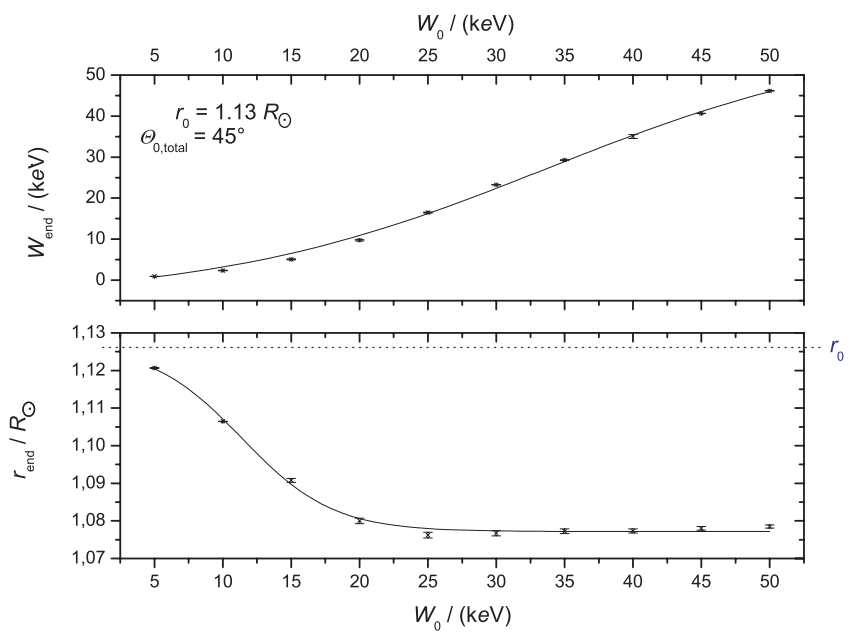

Fig. 10. Influence of the initial energy for the inner transport process. Top: the electron's kinetic energy is illustrated at the point $u_{\|}=0$ depending on its initial kinetic energy. Bottom: the heliocentric radial distance is plotted at the point $u_{\|}=0$ depending on its initial kinetic energy.

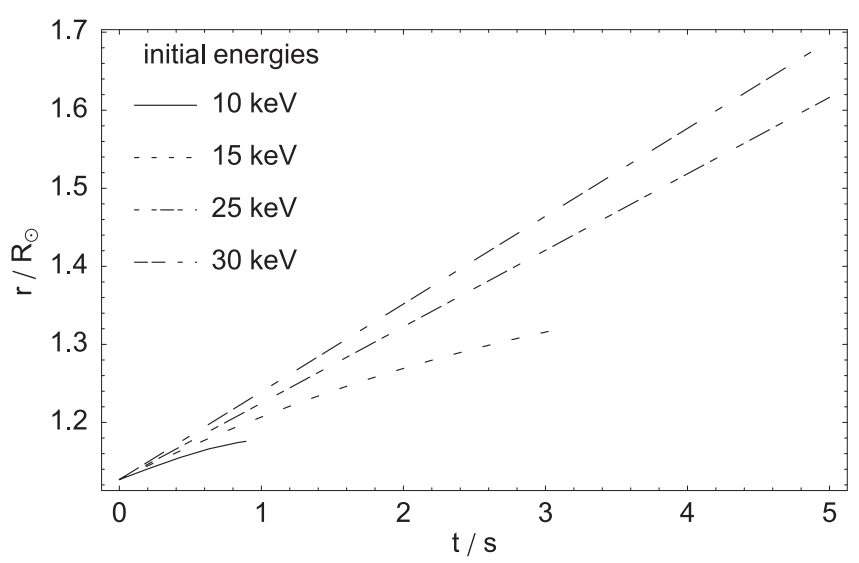

Fig. 11. The electrons' heliocentric altitude is plotted as a function of time. Each line represents the minimum mean square deviation of 1000 calculations depending on the initial electron energy (for pitch angle of $45^{\circ}$, heliocentric altitude of $r_{0} \approx 1.13 R_{\odot}$ ). The procedure for obtaining those fits is analogous to the illustrated procedure in Fig. 8.

\section{Plasma background}

To study the motion of an energetic electron through the solar plasma, it is necessary to know the behaviour of the density as well as the electric and magnetic fields in these regions. It is known that the behaviour of these quantities is different from case to case, since the solar atmosphere is highly variable in its spatial and temporal scales. Nevertheless, the models presented in this section should be regarded only as typical behaviour of these quantities, allowing a quantitative study of the test electron propagation process. They are chosen because of their simplicity and their good agreement with observations.

\subsection{Plasma composition}

First the plasma composition in the solar atmosphere has to be defined. For simplicity's sake, the relative plasma composition in the whole solar atmosphere is considered to be constant. In addition, the solar plasma is assumed to be fully ionised. Thus it is assembled by electrons, protons, and $\mathrm{He}^{2+}$-ions as follows: $N_{\mathrm{e}}=v_{\mathrm{e}} N \approx 0.52 N, N_{\mathrm{p}}=v_{\mathrm{p}} N \approx 0.44 \mathrm{~N}$, and

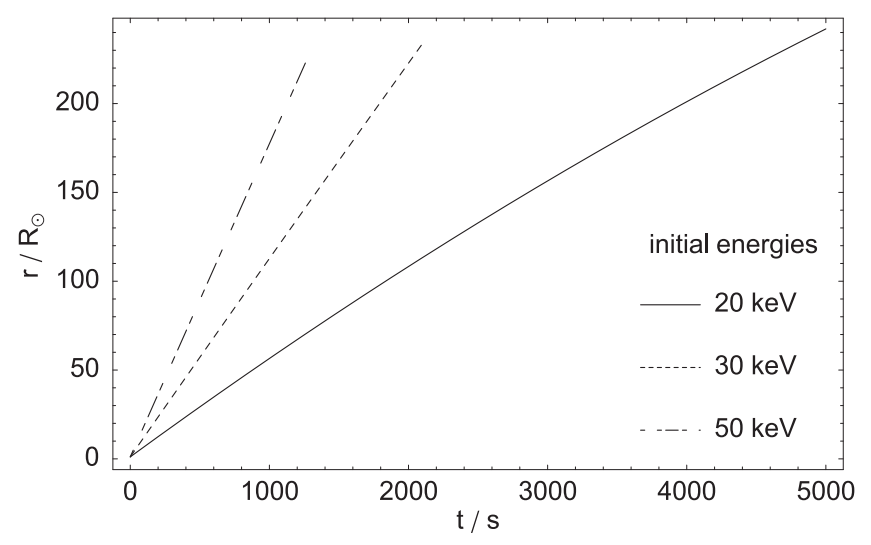

Fig. 12. Same as in Fig. 11, but with a different scale.

$N_{\mathrm{He}^{2+}}=v_{\mathrm{He}^{2+}} N \approx 0.04 N$. Here $N$ denotes the total particle number density, which is the sum of the electron $N_{\mathrm{e}}$, the proton $N_{\mathrm{p}}$, and the helium ion $\left(\mathrm{He}^{2+}\right) N_{\mathrm{He}^{2+}}$ number densities. These specified portions lead to a mean atomic weight of $\rho=0.6$ (see Priest 1984, Chap. 2, p. 82), which is appropriate for the coronal plasma. Thus $N$ is related to the electron number density by $N=v_{\mathrm{e}}^{-1} N_{e} \approx 1.92 N_{\mathrm{e}}$.

\subsection{Plasma density model}

Next it is explained how the plasma density depends on the altitude. The low corona can be regarded as a gravitationally stratified atmosphere. Thus a barometric model given by

$N[r]=N_{\odot} \cdot \exp \left[\xi\left(\frac{R_{\odot}}{r}-1\right)\right] \quad$ and $\quad \xi=\frac{\rho m_{\mathrm{p}} G M_{\odot}}{k_{\mathrm{B}} T R_{\odot}}$

can be applied to the lower corona. Symbols $m_{\mathrm{p}}, G, M_{\odot}, k_{\mathrm{B}}$, $T$, and $R_{\odot}$ represent proton mass, gravitational constant, mass of the Sun, Boltzmann constant, temperature, and solar radius, respectively.

Equation (19) can be deduced from the momentum equation assuming an isothermal and hydrostatic equilibrium (see, e.g., Mann et al. 1999; Önel 2004). This density model agrees very well with the white-light observations of the corona by Koutchmy (1994). Please note that Eq. (19) turns into the $\alpha$-fold Newkirk (1961) model if $N_{\odot}=\alpha 1.68 \times 10^{15} \mathrm{~m}^{-3}$ is used and the temperature is chosen to be $T=1.4 \times 10^{6} \mathrm{~K}$. In this case $\xi$ becomes $\xi=9.95$. The quantity $N_{\odot}$ denotes the full particle number density in the lower corona. The Newkirk parameter $\alpha$ is a free parameter. It makes it poissible to adjust the Newkirk (1961) density model to the present conditions on the Sun (Koutchmy 1994): $\alpha$ is chosen to be 1 in the case of a quiet Sun (very little magnetic activity at the equatorial regions). It is chosen to be 4 in the case of an active Sun, and 10 in the case of the presence of dense coronal loop structures. A moderate choice of $\alpha=4$ for the Newkirk parameter has been made in this paper, since a lot of energetic particles are released in periods of increased solar activity.

The lower corona fits the described barometric, static, and isothermal model of Newkirk (1961) quite well. On the other hand, it is well known that a steady flow of plasma continuously takes place due to solar wind. Even though the model of Newkirk (1961) is appropriate to describe the density behaviour in the low corona very well, it fails to describe the observed densities in the high corona. Therefore, a density model for the high corona and the interplanetary medium, which considers the solar wind, 


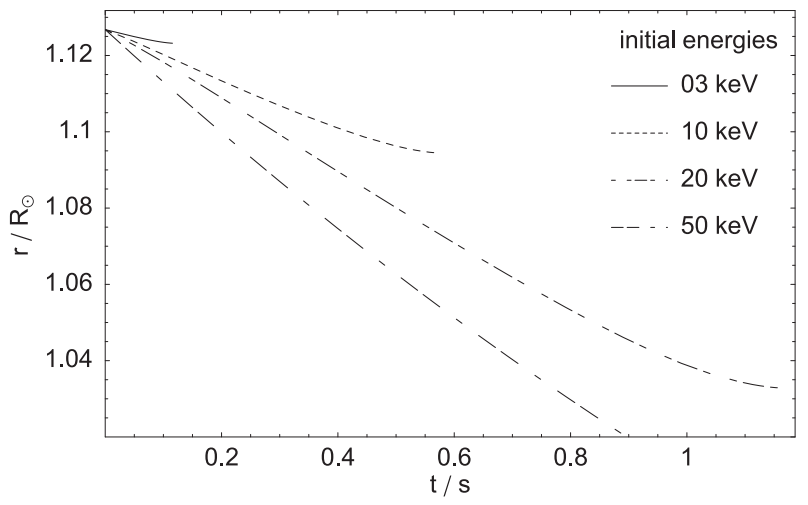

Fig. 13. Same as in Fig. 11, but for inward transport.

is needed. Such a model can be found in terms of a special solution of the wind equation developed by Parker (1981)

$\frac{u_{\mathrm{W}}}{u_{\mathrm{c}}^{2}} \frac{\mathrm{d} u_{\mathrm{W}}}{\mathrm{d} r}=-\frac{1}{N} \frac{\mathrm{d} N}{\mathrm{~d} r}-2 \frac{r_{\mathrm{c}}}{r^{2}}$.

Here $u_{\mathrm{W}}$ stands for the flow (solar wind) velocity. The abbreviations $r_{\mathrm{c}}:=0.5 G M_{\odot} u_{\mathrm{c}}^{-2} \approx 4.15 R_{\odot}$ and $u_{\mathrm{c}}:=\left(k_{\mathrm{B}} T \rho^{-1} m_{\mathrm{p}}^{-1}\right)^{0.5} \approx$ $117 \mathrm{~km} \mathrm{~s}^{-1}$ give the critical distance and velocity of the solar wind, respectively, when it becomes supersonic. Mann et al. (1999) presented a solution of Eq. (20) for a temperature of $T=10^{6} \mathrm{~K}$. They obtained a heliospheric density model by adding the equation of continuity

$N[r] \cdot u_{\mathrm{W}}[r] \cdot r^{2}=C_{\mathrm{P}}$,

where the constant $C_{\mathrm{P}}$ has been determined from observations of the particle flux at $1 \mathrm{AU} C_{\mathrm{P}}=1.21 \times 10^{34} \mathrm{~s}^{-1}$ (Schwenn 1990). This solution for the density model is illustrated in Fig. 3. It is in good agreement with the observed densities in the heliosphere up to a radial distance of 5 AU (Mann et al. 1999).

After the density models for the lower and the higher atmosphere have been introduced separately, both of them shall be united in one global model for the atmosphere. This global model obtained from the combination between both previously introduced models is used in the present paper for the numerical calculations. The combination of both models is simply performed by using

$N[r]=\left\{\begin{array}{c}N_{\text {Newkirk }}[r, \alpha=4] \\ N_{\text {Mann }}[r]\end{array}\right.$ for $\begin{array}{l}r<r_{\mathrm{S}} \\ r \geq r_{\mathrm{S}}\end{array}$.

Here $r_{\mathrm{S}}$ is the point of intersection between the Newkirk (1961) model, to which the index "Newkirk" refers, and the model of Mann et al. (1999), to which the index "Mann" refers. Note that $r_{\mathrm{S}}$ is a function of $\alpha$, as it can be easily seen in Fig. 3 (i.e., $r_{\mathrm{s}}=1.86 R_{\odot}, 1.40 R_{\odot}, 1.12 R_{\odot}$ for $\alpha=1,2$, 4, respectively). As a consequence of Eq. (22), the temperature is assumed to behave according to the density models as

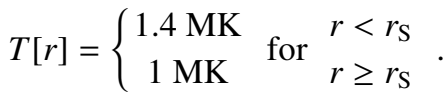

\subsection{Model for the global electric field}

The test electron is also influenced by a global electric field during its propagation along the magnetic field. Such a field is established in a gravitationally stratified atmosphere due to the large

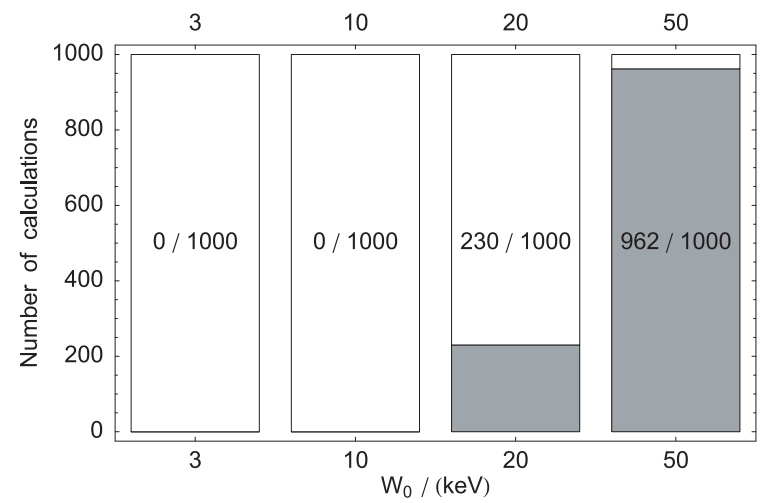

Fig. 14. Histogram showing the number of the electrons, which manage to penetrate down to an altitude of $0.03 R_{\odot}$ above the photosphere, or less. The initial kinetic energy of the electron has been varied from $3 \mathrm{keV}$ up to $50 \mathrm{keV}$, and 1000 calculations have been made for each chosen energy.

mass ratio between protons and electrons. This electric field $E$ can be deduced by the momentum equation of an ideal, isothermal electron fluid

$-e E N_{\mathrm{e}}-k_{\mathrm{B}} T \frac{\mathrm{d} N_{\mathrm{e}}}{\mathrm{d} r}=0$

If the mass of electron is neglected

$E[r]=-\frac{k_{\mathrm{B}} T}{e} \cdot \frac{1}{N[r]} \frac{\mathrm{d} N[r]}{\mathrm{d} r}$

is obtained. Adopting the density model given in Eq. (22), the radial behaviour of the global electric field can be determined as depicted in Fig. 4. The chosen temperature $T$ follows from Eq. (23). Note that in this case the electric field vector is assumed to point towards the Sun and the electrostatic potential $\Phi$ can be evaluated between the radial distance $r_{1}$ and $r_{2}$ from the centre of the Sun

$-\int_{r_{1}}^{r_{2}} \mathrm{~d} r[E]=\Phi\left[r_{2}\right]-\Phi\left[r_{1}\right]=\Delta \Phi=\frac{k_{\mathrm{B}} T}{e} \cdot \ln \left|\frac{N\left[r_{2}\right]}{N\left[r_{1}\right]}\right|$.

For instance, there is an electrostatic potential difference $\Delta \Phi$ of about $137 \mathrm{~V}$ between the bottom of the corona at $r \approx R_{\odot}$, i.e., slightly above the photosphere and the $300 \mathrm{MHz}$ electron plasma frequency level, which (according to Eq. (22) with $\alpha=4$ ) corresponds to a height of about $88 \mathrm{Mm}$ above the photosphere. On the other hand, the potential difference is $1.63 \mathrm{kV}$ between the $300 \mathrm{MHz}$ level and $1 \mathrm{AU}$ according to the density model introduced above.

\subsection{Global magnetic field}

To describe the coronal magnetic flux density $B$, in the distance range between $1.02 R_{\odot}$ and $10 R_{\odot}$, the empiric model by Dulk \& McLean (1978) has been chosen

$B_{\mathrm{D} \& \mathrm{M}}[r]=0.5 \cdot\left(\frac{r}{R_{\odot}}-1\right)^{-1.5} \times 10^{-4} \mathrm{~T}$.

The index "D\&M" refers to the model of Dulk \& McLean (1978). On the other hand, by analysing in situ measurements of HELIOS 1 and 2 Mariani \& Neubauer (1990) have found that $B$ decreases above $10 R_{\odot}$ with

$B_{\mathrm{M} \& \mathrm{~N}}[r]=\sqrt{\left(\frac{\sqrt{C_{\mathrm{r}}}}{r^{2}}\right)^{2}+\left(\frac{\sqrt{C_{\phi}}}{r^{1.1}}\right)^{2}}$ 
which is close to the predictions of Parker (1958). The index "M\&N" refers to the model of Mariani \& Neubauer (1990). The constants $C_{\mathrm{r}} \approx 7.99 \mathrm{~T}^{2} \mathrm{~m}^{4}$ and $C_{\phi} \approx 9.61 \times 10^{7} \mathrm{~T}^{2} \mathrm{~m}^{2.2}$ follow, if $B_{\phi}[1 \mathrm{AU}]=\left(C_{\phi}\right)^{0.5} \cdot(1 \mathrm{AU})^{-1.1}=5 \mathrm{nT}$ (Musmann et al. $1977)$ is taken into account, and

$B[r]=\left\{\begin{array}{l}B_{\mathrm{D} \& \mathrm{M}}[r] \\ B_{\mathrm{M} \& \mathrm{~N}}[r]\end{array}\right.$ for $\begin{array}{c}1.02 R_{\odot} \lesssim r \leq 10 R_{\odot} \\ 10 R_{\odot} \leq r \lesssim 1 \mathrm{AU}\end{array}$

is assumed. Equation (29) describes the behaviour of the radial global magnetic field. It is illustrated in Fig. 5.

At this point, the condition stated in Eq. (2) shall be investigated. According to that condition, the magnetic moment will only be an adiabatic constant of motion, if $\mathcal{B} \ll 1$ is fulfilled. To verify this relation, $\mathcal{B} \sim r_{\text {Larmor }} \sim \sqrt{W} \sin [\Theta]$ is used, and the largest $\mathcal{B}$ possible is estimated. ${ }^{1}$ For $50 \mathrm{keV}$ electrons with a pitch angle of $\Theta=90^{\circ}$ the magnetic field of Eq. (29) fulfils the condition $\mathcal{B} \leq 1.2 \times 10^{-6} \ll 1$, within the whole distance range from $1.02 R_{\odot}$ to $1 \mathrm{AU}$. The Larmor radius varies from $r_{\text {Larmor }}\left[1.02 R_{\odot}\right]=4.3 \times 10^{-2} \mathrm{~m}$ to $r_{\text {Larmor }}[1 \mathrm{AU}]=1.2 \times 10^{5} \mathrm{~m}$.

Note, that the electric field is considered to be aligned parallelly to the magnetic field. The introduced magnetic model does not take Parker's spiral into account. The direction of the magnetic field line is not of major importance for the electron motion, but the magnetic field strength is, because the electron is guided along the magnetic field, and the direction of the gyromotion does not influence its drift motion (Alfvén's gyro centre approximation, Eqs. (6) and (7)). After introducing the radial behaviour of the density and the temperature as well as the global electric and magnetic field, the conditions of the background plasma are well described for studying the propagation of energetic electrons in the corona and interplanetary space numerically. That is done in the next section.

\section{Numerical treatment and discussions}

In this paper only the propagation process itself is discussed, the electron acceleration mechanisms are not treated. Thus the only inputs needed for the numerical studies are the initial energy and the initial height of the test electron, if the complete parameters of the plasma background (see Sect. 3), i.e., models for the particle density Eq. (22), the electric field Eq. (25), and the magnetic field Eq. (29) are known. Then the Eqs. (16) to (18) can be solved numerically by iteration. The physical constants needed for that are taken from the CODATA recommendation (Mohr \& Taylor 2005). The results of the numerical evaluation of the model presented in the previous sections are discussed in the following.

According to the type III radio burst observations, the acceleration site is assumed to be located near the $300 \mathrm{MHz}$ level (e.g., see Fig. 2). Thus this electron plasma frequency is chosen in correspondence to the test electron's initial heliocentric altitude of $r_{0} \approx 1.13 R_{\odot}$, with the density model (see Eq. (22) for $\alpha=4$, as in Fig. 3) used. At first a test electron with an initial energy of $20 \mathrm{keV}$ and an initial pitch angle of $45^{\circ}$ is considered. In the beginning, the motion towards the Sun is regarded (Fig. 6). Due to the electron's high initial energy, the effect of the global electric field, which acts in the form of an acceleration on it, is very weak, since the potential difference between the height of $0.13 R_{\odot}$ and the bottom of the corona is about $137 \mathrm{~V}$. On the other hand, the influence of the increasing magnetic field

\footnotetext{
1 According to Eq. (3) the Larmor radius is related to the electron's kinetic energy $W=0.5 m_{\mathrm{e}} u^{2}$ by $r_{\text {Larmor }} \sim u \sin [\Theta] \sim \sqrt{W} \sin [\Theta]$.
}

leads to a the mirror force acting outward, i.e., against the inward motion. Thus, the drift energy (Fig. 6, bottom right panel) is dramatically transferred into the Larmor energy accompanied with a monotonic increase of the pitch angle (Fig. 6, bottom left panel). Here, the drift energy and Larmor energy are defined as the kinetic energy with respect to the motion parallel and perpendicular to the magnetic field, respectively. The amount of the total energy (as the sum of the drift and Larmor energy) is weakly decreasing due to the influence of the Coulomb collisions (Fig. 6). The action of the Coulomb collisions is also seen in the scatter-like increase of the pitch angle. After $0.3 \mathrm{~s}$ the electron reaches a height of $0.105 R_{\odot}$ above the photosphere (Fig. 6, top left panel). This height corresponds to an electron plasma frequency $^{2}$ of $335 \mathrm{MHz}$ (Fig. 6, top left panel). Thus, the electron would cause a reverse drifting type III radio burst with a drift rate of $117 \mathrm{MHz} \mathrm{s}^{-1}$, i.e., a positive drift rate, which means that the burst drifts towards higher frequencies.

As already mentioned, the Coulomb collisions cause a random motion of the electron. A test electron with an initial energy of $20 \mathrm{keV}$ and an initial pitch angle of $45^{\circ}$ starts at a height of $0.13 R_{\odot}$ above the photosphere, to illustrate that behaviour. Each repetition of this calculation (1000 times in total) gives a random walk of such a test electron. They are depicted in Fig. 8 together with the best fit of the 1000 electron trajectories, according to the minimum mean square deviation. Even if throughout all of these calculations the same initial conditions are used, 1000 different solutions are obtained for each run. It can clearly be seen that there are electron trajectories, which indicate that the test electrons stop at different heights for each performed calculation. This kind of behaviour follows from the binary dice, which was introduced in Eq. (11) to treat the Coulomb collisions correctly.

The motion of electrons with different initial energies, but the same initial pitch angles of $45^{\circ}$ are considered in Fig. 13. It can be seen there that the electron with an initial energy of $3 \mathrm{keV}$ already stops after $0.1 \mathrm{~s}$. Those electrons with initial energies greater than $20 \mathrm{keV}$ are able to penetrate deep into the low corona within $1 \mathrm{~s}$.

A similar study but for the outer motion is presented for the corona in Fig. 11 and for the interplanetary space in Fig. 12. Here, all electrons have an initial pitch angle of $45^{\circ}$ again. The electron with the initial energy of $10 \mathrm{keV}$ already stops in the corona after $1 \mathrm{~s}$. Electrons with an initial energy higher than $20 \mathrm{keV}$ are able to leave the corona and reach the distance of 1 AU after few $1000 \mathrm{~s}$ (Fig. 12). As expected, the electrons with a higher initial energy reach 1 AU more quickly than the ones with a low initial energy.

Next the outer motion (i.e., toward the interplanetary space) is considered for an electron with an initial energy of $30 \mathrm{keV}$ and an initial pitch angle of $45^{\circ}$ (Fig. 7). Here, it starts again at the $300 \mathrm{MHz}$ level $\left(r_{0} \approx 1.13 R_{\odot}\right)$ and reaches the $50 \mathrm{MHz}$ level at a height of $0.6 R_{\odot}$ above the photosphere after approximately $4 \mathrm{~s}$. This motion would cause a type III radio burst with a drift rate of $-63 \mathrm{MHz} \mathrm{s}^{-1}$, which is a reasonable type III drift rate (Mann \& Klassen 2002). Because of the decreasing global magnetic field, the Larmor energy is dramatically converted into drift energy (Fig. 7, bottom right panel). Due to Coulomb collisions the electron loses about $23 \%$ of its initial energy during its motion (Fig. 7). Because the electrostatic potential between $1.13 R_{\odot}$ and $1 \mathrm{AU}$ is about $1.6 \mathrm{keV}$ and due to the decreasing particle density of the atmosphere and its high drift energy, this electron is able to leave the solar atmosphere, with just a little loss of

\footnotetext{
2 The electron plasma frequency is defined by $f=\sqrt{\frac{e^{2} N_{\mathrm{e}}}{4 \pi^{2} \epsilon_{0} m_{\mathrm{e}}}}$ (see Treumann \& Baumjohann 1999, Chap. 9, p. 202).
} 
energy. It reaches the distance of 1 AU from the centre of the Sun after $2000 \mathrm{~s}$ (see Fig. 12) with a final energy of $23 \mathrm{keV}$ (Fig. 7, bottom right panel).

To study the dependence of the penetration depth on the initial pitch angle of an electron, a test electron with an initial energy of $20 \mathrm{keV}$ is considered to start at a height of $0.13 R_{\odot}$ above the photosphere. The initial pitch angle varys between $5^{\circ}$ and $85^{\circ}$. As expected, an electron with a small initial pitch angle can penetrate deeper into the lower corona than those with higher initial pitch angles (Fig. 9). But they lose a large amount of energy due to Coulomb collisions, as shown in Fig. 9. For instance, an electron with an initial pitch angle of $5^{\circ}$ is able to penetrate down up to a height of $0.05 R_{\odot}$, while it loses energy from initially $20 \mathrm{keV}$ down to $5 \mathrm{keV}$ at the end (see Fig. 9).

In addition, the dependence of the penetration depth and the final energy from the initial one is demonstrated in Fig. 10, where the initial pitch angle is chosen to be $45^{\circ}$ again, for instance. As expected, electrons with smaller initial energy lose much more energy due to Coulomb collisions than those of higher initial energies. On the other hand, electrons with an initial energy higher than $20 \mathrm{keV}$ are able to penetrate substantially deep into the low corona, i.e., down to $0.078 R_{\odot}$ above the photosphere (Fig. 10).

In one further attempt, the penetration depth (inner transport) is calculated for electrons starting with an initial pitch angle of $0^{\circ}$ at an initial altitude over the photosphere of $0.13 R_{\odot}$, where the $300 \mathrm{MHz}$ level is expected. Due to the chosen pitch angle, there is no gyro motion for the electrons in these calculations and the Larmor energy vanishes, while the total kinetic energy is represented by the drift energy only. The initial drift energy has been varied from $3 \mathrm{keV}$ up to $50 \mathrm{keV}$. This study answered the question of how many electrons have been able to reach an altitude of $0.03 R_{\odot}$ above the photosphere or less. Its results can be seen in Fig. 14. The number of those electrons that have been able to penetrate through the solar corona toward the photosphere increased dramatically with the increasing energy. But even in those calculations where the initial energy has been set to $50 \mathrm{keV}, 3.8 \%$ of 1000 electrons have not been able to reach a height less than or equal to $0.03 R_{\odot}$ above the photosphere due to Coulomb collisions.

\section{Summary}

In the present paper a model for energetic electron propagation through a given plasma background with coronal and interplanetary properties has been introduced and numerically evaluated. The influence of the global magnetic and electric fields on the one hand and of the Coulomb collisions on the motion of energetic electrons on the other hand are quantitatively discussed by varying the initial conditions of the electrons (see Figs. 6 to 12), whereas the plasma background as introduced Sect. 3 is considered to be fixed for this study.

The major improvement of the deduced model is the new way of treating the effects of the Coulomb scattering process on the pitch angle by introducing a binary dice Eq. (11). We are not aware of any other transport model (e.g., Brown 1972; Bai 1982; Emslie 1978; Leach \& Petrosian 1981; Lu \& Petrosian 1988; Estel \& Mann 1999), that has considered the influence of the Coulomb scattering process on the pitch angle.

The presented transport model allows investigation of the electron energies along their trajectories, and it is based on observations of the electrons traversing the coronal and interplanetary plasma. That makes it poissible to locate the altitude of the acceleration site by means of reverse modelling, when a sufficient number of electrons propagating inwards and outwards is considered (see Sect. 2.3). In the case of the electrons travelling inward, it is sufficient to observe the locations and sources of the chromospheric X-ray radiation, whereas this information for the electrons travelling outwards is measured in situ.

Acknowledgements. The authors want to thank H. Blersch and his team from the computer room of the Physics Department at the University of Technology Berlin, which supported our work with computer power. Furthermore, the authors would like to express their thanks to M. Skender and R. Miteva for their many helpful comments. We also appreciate the anonymous referee, whose skilled remarks helped to improve this paper. Last but not least, we thank our language editor E. Buzay.

\section{Appendix A: Mean squared scattering angle}

The scattering angle $\theta$ is given (see Appendix B1, p. 312 in Treumann \& Baumjohann 1999; Chap. 6, p. 290 in Krall \& Trivelpiece 1986) in the centre of the mass system by

$\theta=2 \arctan \left[\frac{-e q}{4 \pi \epsilon_{0}} \cdot \frac{1}{\chi b u^{2}}\right]$,

where $b$ is the impact parameter. Then the mean squared scattering angle $\overline{\theta^{2}}$ follows from

$\mathrm{d} \overline{\theta^{2}}=\int_{b_{0}}^{\lambda_{\mathrm{D}}} \mathrm{d} b\left[\theta^{2} \cdot N 2 \pi b u \mathrm{~d} t\right]$

because $\mathrm{d} N=N 2 \pi \mathrm{d} b u \mathrm{~d} t$ represents the number of Coulomb collisions (see Krall \& Trivelpiece 1986, Chap. 6, p. 292), where each collision leads to a deflection of $\theta$, if the test electron passes through the distance $u \mathrm{~d} t$. The exact solution of Eq. (A.2) (as given in Önel 2004) is

$$
\begin{aligned}
\frac{\mathrm{d} \overline{\theta^{2}}}{\mathrm{~d} t}=\mid & \frac{e^{2} q^{2} N}{2 \pi \epsilon_{0}^{2} \chi^{2} u^{3}} \ln \left[\sqrt{\frac{\lambda_{\mathrm{D}}^{2}+b_{0}^{2}}{2 b_{0}^{2}}}\right] \\
& +\frac{e^{2} q^{2} N}{4 \pi \epsilon_{0}^{2} \chi^{2} u^{3}} \arctan ^{-2}\left[\frac{\lambda_{\mathrm{D}}}{b_{0}}\right] \\
& +4 \pi \lambda_{\mathrm{D}}^{2} u \arctan ^{-2}\left[\frac{\lambda_{\mathrm{D}}}{b_{0}}\right] \\
& +2 \frac{-e q N \lambda_{\mathrm{D}}}{\epsilon_{0} u \chi} \arctan ^{-1}\left[\frac{\lambda_{\mathrm{D}}}{b_{0}}\right] \\
& -\frac{e^{2} q^{2} N}{8 \epsilon_{0}^{2} \chi^{2} u^{3}}(\pi+1) \mid .
\end{aligned}
$$

Equation (A.3) can be simplified by assuming only small angle scattering processes, which are reasonable for the corona and the interplanetary medium (Estel \& Mann 1999; Estel 1999). This assumption makes Eq. (A.1) turn into

$$
\theta \approx \frac{-e q}{2 \pi \epsilon_{0}} \cdot \frac{1}{\chi b u^{2}}
$$

With Eqs. (A.2) and (A.4) the known (Jackson 1975, Chap. 13, p. 649) simplified expression of Eq. (A.3), namely Eq. (10)

$$
\frac{\mathrm{d} \overline{\theta^{2}}}{\mathrm{~d} t} \approx\left|\frac{e^{2} q^{2} N}{2 \pi \epsilon_{0}^{2} \chi^{2} u^{3}} \ln \left[\sqrt{\frac{\lambda_{\mathrm{D}}^{2}+b_{0}^{2}}{2 b_{0}^{2}}}\right\rfloor\right|
$$

is obtained. 


\section{References}

Bai, T. 1982, ApJ, 259, 341

Brown, J. C. 1971, Sol. Phys., 18, 489

Brown, J. C. 1972, Sol. Phys., 26, 441

Dulk, G. A., \& McLean, D. J. 1978, Sol. Phys., 57, 279

Emslie, A. G. 1978, ApJ, 224, 241

Estel, C. 1999, Ph.D. Thesis, Universität Potsdam, Germany

Estel, C., \& Mann, G. 1999, A\&A, 345, 276

Goldston, R. J., \& Rutherford, P. H. 1995, Introduction to Plasma Physics (Institute of Physics Publishing)

Jackson, J. D. 1975, Classical Electrodynamics (John Wiley \& Sons, Inc.)

Kegel, W. H. 1998, Plasmaphysik, Eine Einführung, X, 320 S. 61 Abb., 5 in Farbe. (Berlin, Heidelberg, New York: Springer-Verlag)

Koutchmy, S. 1994, Adv. Space Res., 14, 29

Krall, N. A., \& Trivelpiece, A. W. 1986, Principles of Plasma Physics (San Francisco Press, Inc.)

Kunow, H., Wibberenz, G., Green, G., Müller-Mellin, R., \& Kallenrode, M.-B. 1991, in Physics and Chemistry in Space, Physics of the Inner Heliosphere II: Particles, Waves and Turbulence, ed. R. Schwenn, \& E. Marsch (Heidelberg, Berlin: Springer-Verlag), 21, 243

Leach, J., \& Petrosian, V. 1981, ApJ, 251, 781

Lin, R. P. 1974, Space Sci. Rev., 16, 189

Lin, R. P., Dennis, B. R., Hurford, G. J., et al. 2002, Sol. Phys., 210, 3

Lu, E. T., \& Petrosian, V. 1988, ApJ, 327, 405

Mann, G., \& Klassen, A. 2002, in Solar Variability: From Core to Outer Frontiers, ed. J. Kuijpers, ESA SP-506, 245

Mann, G., Aurass, H., Voigt, W., \& Paschke, J. 1992, in ESA SP-348: Coronal Streamers, Coronal Loops, and Coronal and Solar Wind Composition, ed. C. Mattok, ESA SP-348, 129

Mann, G., Jansen, F., MacDowall, R. J., Kaiser, M. L., \& Stone, R. G. 1999, A\&A, 348, 614
Mariani, F., \& Neubauer, F. M. 1990, in Physics and Chemistry in Space, Physics of the Inner Heliosphere I: Large-Scale Phenomena, ed. R. Schwenn, \& E. Marsch (Heidelberg, Berlin: Springer-Verlag), 20, 183

Melrose, D. B. 1985, in Solar Radiophysics: Studies of Emission from the Sun at Metre Wavelengths, ed. D. J. McLean, \& N. R. Labrum (Cambridge: Cambridge University Press), 177

Mohr, P. J., \& Taylor, B. N. 2005, Rev. Mod. Phys., 77, 1

Musmann, G., Neubauer, F. M., \& Lammers, E. 1977, JGZG, 42, 591

Newkirk, G. J. 1961, ApJ, 133, 983

Önel, H. 2004, Diploma thesis, University of Technology Berlin in collaboration with the Astrophysical Institute Potsdam, Germany

Önel, H., Mann, G., \& Sedlmayr, E. 2005, in The Dynamic Sun: Challenges for Theory and Observations, ESA SP-600

Önel, H., Mann, G., \& Sedlmayr, E. 2006, in Planetary Radio Emissions VI

Parker, E. N. 1958, ApJ, 128, 664

Parker, E. N. 1981, ApJ, 251, 266

Priest, E. R. 1984, Solar Magneto-hydrodynamics (Dordrecht, Holland: D. Reidel Publishing Company)

Robinson, R. D. 1985, in Solar Radiophysics: Studies of Emission from the Sun at Metre Wavelengths, ed. D. J. McLean, \& N. R. Labrum (Cambridge: Cambridge University Press), 385

Schwenn, R. 1990, Large-Scale Structure of the Interplanetary Medium (Physics of the Inner Heliosphere I), 99

Treumann, R. A., \& Baumjohann, W. 1997, Adv. Space Plasma Phys. (Imperial Colleage Press)

Treumann, R. A., \& Baumjohann, W. 1999, Basic Space Plasma Phys. (Imperial Colleage Press)

Yokoyama, T., Nakajima, H., Shibasaki, K., Melnikov, V. F., \& Stepanov, A. V. 2002, ApJ, 576, L87 